\title{
Asentamientos irregulares, vivienda precaria y población vulnerable al clima en la ciudad de Hermosillo, Sonora: un estudio exploratorio
}

\author{
Nicolás Pineda Pablos, Alejandro Salazar Adams, América Lutz Ley, \\ Luis Miguel Silva Gutiérrez, América Murrieta Saldivar*
}

\section{Introducción}

La adaptación de la ciudad de Hermosillo al medio natural que la rodea, caracterizado por la exposición periódica a altas temperaturas, implica contar con mecanismos de previsión y mitigación de los efectos de las temperaturas extremas sin menoscabo del desarrollo de las actividades económicas ni de la calidad de vida (Varady, Scott, Wilder, Morehouse, Pineda, \& Garfin, 2013).

En este reto, la población más vulnerable es la que habita en viviendas precarias que carecen de los medios para mitigar el calor o para evitar las deshidrataciones, golpes de calor, diarreas e incluso la muerte. Dentro de la población que habita en estas viviendas, los grupos más vulnerables son los niños, las personas de la tercera edad y las mujeres que permanecen la mayor parte del tiempo en dichas viviendas.

El presente trabajo muestra los resultados de un estudio llevado a cabo en la ciudad de Hermosillo, Sonora, con el objetivo de explorar el problema de la población vulnerable

\footnotetext{
"Profesor de la Universidad de Sonora e investigador de El Colegio de Sonora, npineda@colson.edu.mx; Investigador de El Colegio de Sonora, asalazar@colson.edu.mx; Estudiante de doctorado, Universidad de Arizona, americalutz@gmail.com; Maestro en Ciencia Sociales, El Colegio de Sonora, miguelsilvagtz@gmail.com; Maestra en Ciencias Sociales, El Colegio de Sonora, amurrieta@colson. edu.mx

${ }^{6}$ El estudio es parte del proyecto "Flujos de información y políticas públicas", financiado por el Udall Center de la Universidad de Arizona y el Inter-American Institute for Global Change Research (IAI).
}

al clima, principalmente al calor extremo. ${ }^{6} \mathrm{La}$ decisión de estudiar los asentamientos irregulares en la ciudad de Hermosillo obedece a que el estatus de irregularidad de la vivienda generalmente va asociado a un estado precario de la misma, y por lo tanto, expuesto a los elementos ambientales como el calor. La historia de estas invasiones es la contraparte y el subproducto de lo que se ha llamado la ciudad de la revolución o de los megaproyectos (Méndez, 2000), es decir, de los gobernantes emanados de la Revolución Mexicana y del Partido Revolucionario Institucional (Méndez, 2000).

\section{Asentamientos irregulares y vivienda precaria}

Los asentamientos irregulares, identificados localmente como "invasiones", se comenzaron a dar a causa del crecimiento explosivo de la ciudad a partir de los años cincuenta. La primera regularización colectiva registrada en la época reciente fue la de la colonia El Choyal que surgió de una reubicación que hizo en 1965 el gobernador Luis Encinas de los habitantes asentados en el vado del río Sonora y que, por los riesgos de una inundación debido al crecimiento del río, fue obligada a reubicarse (Gutiérrez, 1994:24).

Se agradece además, la cooperación y los apoyos recibidos de la Sindicatura Municipal de Hermosillo 2009-2012, presidida por Luis Enrique Terrazas Romero. 
La lista histórica de las invasiones que ha regularizado la Sindicatura del gobierno municipal de Hermosillo (López, 2011), incluye los siguientes antecedentes de invasiones:

En 1972 surgen los asentamientos irregulares de las colonias Ley 57, Benito Juárez y Francisco Villa, en el norte de la ciudad (véase también: Lagarda, 2008).

En 1974 aparecen las invasiones de las colonias López Portillo y López Mateos, al norte del anillo periférico en el norte de la ciudad. En estos años surge también la invasión de la colonia Café Combate al oriente.

En 1976 surge la invasión de la colonia Emiliano Zapata.

1980 se hace la invasión de la colonia Lomas de Madrid, también al norte.

1981 surge la invasión Mártires de Cananea y la actual colonia Primero Hermosillo.

1985 se hace la invasión Miguel Hidalgo.

Inicialmente las invasiones se componen exclusivamente de viviendas construidas con materiales precarios, sin embargo, a medida que pasa el tiempo y avanza su proceso de regularización y titulación, las viviendas precarias se van transformando, por medio de la autoconstrucción, en viviendas formales.

Un problema para censar las invasiones fue que, en el año 2010, existían cuatro dependencias públicas relacionadas con los procesos de regularización del suelo urbano:

Sindicatura del Ayuntamiento de Hermosillo: es la responsable del suelo urbano de la ciudad, lleva la mayoría de los procesos legales de los invasores. En este caso, se obtuvo información tanto de las colonias que actualmente se encuentran en proceso de negociación o de legalización, como de las que históricamente fueron invasiones, pero ya fueron regularizadas.

Promotora Inmobiliaria del Municipio de Hermosillo: técnicamente está encargada de la construcción de viviendas para familias de bajos ingresos, pero que en la práctica también desarrolla esta tarea.

Oficina de Bienes y Concesiones del Gobierno del Estado: 1leva los casos que tienen relación con el patrimonio inmobiliario del gobierno del estado.

Comisión para la Regularización de la Tenencia de la Tierra (CORETT): dependencia del gobierno federal que lleva casos de terrenos ejidales.

Un primer conteo de asentamientos irregulares nos da que en el año 2010 existían en Hermosillo 70 barrios urbanos considerados invasiones que tienen como su origen un asentamiento irregular. De estos, para su regularización o titulación, 23 están a cargo de Sindicatura, 11 de Promotora Inmobiliaria, 29 corresponden a Bienes y Concesiones y 9 colonias están a cargo de la CORETT.

A esta cantidad de invasiones de 2010, hay que agregar además, las colonias que fueron regularizadas por gobiernos anteriores. De este tipo tenemos que son 87 las colonias regularizadas por Sindicatura y 26 por Promotora Inmobiliaria. No contamos con los datos de las colonias formalizadas en el pasado por Bienes y Concesiones y por la CORETT. Del total de colonias de Hermosillo, históricamente al menos 185 son colonias que se originaron en asentamientos irregulares. La lista completa de las invasiones de Hermosillo se presenta en el anexo 1.

Cuadro 1. Asentamientos irregulares de Hermosillo

\begin{tabular}{lccc}
\hline Dependencia & $\begin{array}{c}\text { Actuales (en proceso de } \\
\text { regularización) }\end{array}$ & Históricas (regularizadas) & Total \\
\hline Sindicatura & 23 & 87 & 110 \\
\hline Promotora Inmobiliaria & 11 & 26 & 37 \\
\hline Bienes y Concesiones & 29 & n.d. & 29 \\
\hline CORETT & 9 & n.d. & 9 \\
\hline Total & 72 & 113 & 185 \\
\hline
\end{tabular}

Fuente: elaboración propia con base en la lista proporcionada por la Sindicatura Municipal, en abril de 2010.

Con respecto a las 72 invasiones que se encontraban en proceso de regularización en 2010, contamos con información sobre las 34 que se encuentran a cargo de Sindicatura y de Promotora Inmobiliaria del Gobierno Municipal de Hermosillo. Estas 34 invasiones albergan un total de 6104 familias, lo que multiplicado por un índice aproximado de cuatro personas por familias, nos da un total de 24416 habitantes de estas invasiones. La invasión más poblada es la López Mateos en la que se estima viven 1200 familias, mientras que la más pequeña es La Alborada (Ladrilleras) que solo tiene 10 familias (López, 2010). En promedio, estas invasiones tienen aproximadamente 191 familias cada una.

En lo que respecta a su antigüedad, tenemos que la invasión más antigua es la Francisco Villa, que se originó en 1980; y la más reciente es la Guayacán que tuvo lugar en 2008. La antigüedad de las invasiones oscila por lo tanto, entre 30 y 2 años. Además, hay cuatro invasiones sobre las que no se tiene su antigüedad. Las invasiones más recientes son las siguientes:

\section{Savia}




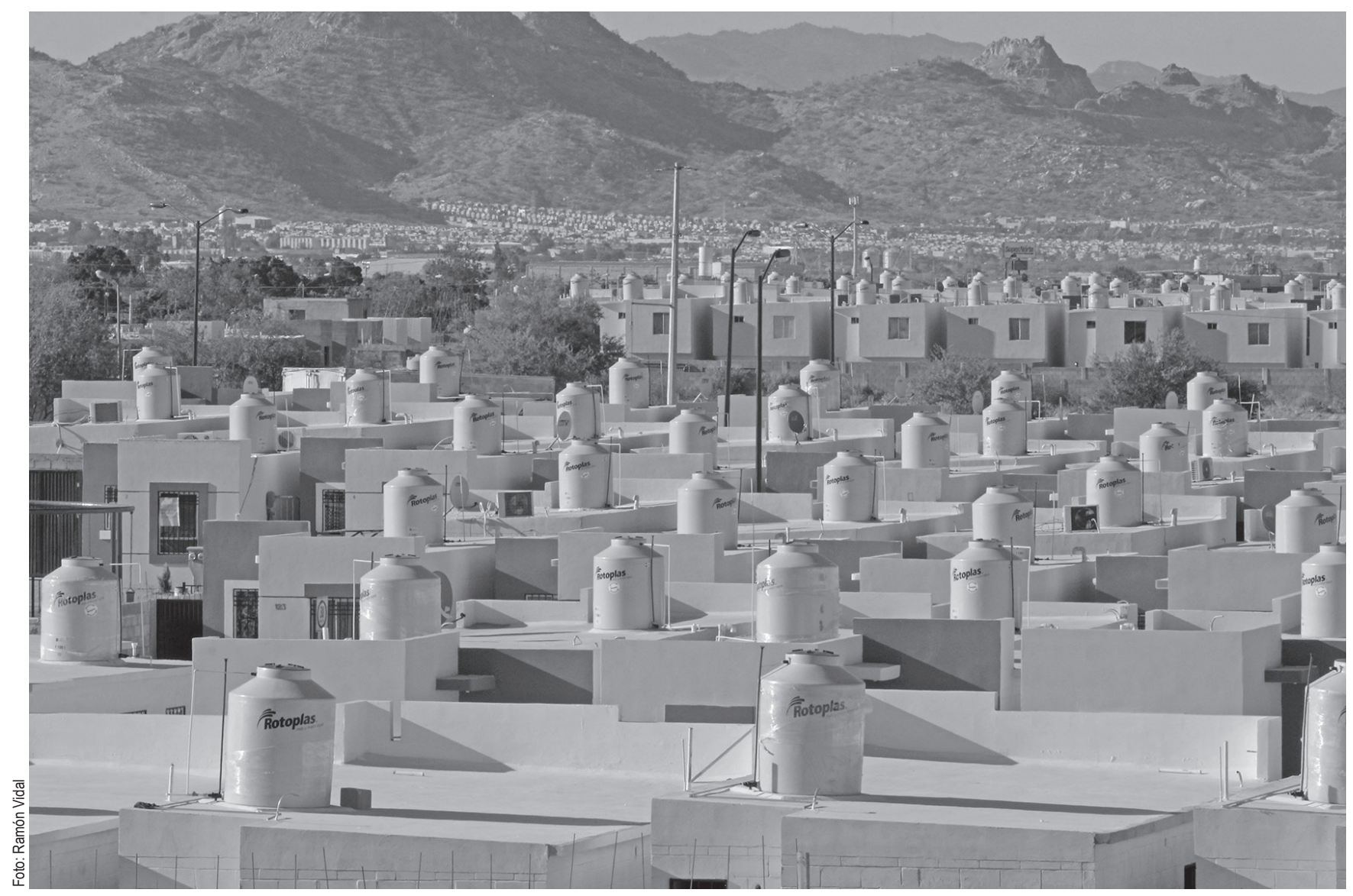

$\begin{array}{ll}\text { Miguel Valencia } & 2002 \\ \text { La Alborada (Las Ladrilleras) } & 2002 \\ \text { La Manga (Sector Costa } & 2003 \\ \text { Solidaridad Occidental } & 2003 \\ \text { El Chaparrral } & 2004 \\ \text { Plutarco Elías Calles } & 2008\end{array}$

Esta lista, nos permite apreciar que dos invasiones corresponden al trienio 2006-2009 (cuando fue presidente municipal Ernesto Gándara), una invasión en el trienio 2003-2006 (siendo presidenta municipal María Dolores del Río) y cuatro invasiones corresponden al trienio de 2000-2003 (cuando era alcalde Francisco Búrquez) (López, 2010).

Las preguntas clave de este estudio son: ¿cuál es la calidad de los materiales de estas viviendas?, ¿están todas construidas con materiales precarios? Con respecto a los materiales de estas viviendas, contamos con la información del estudio realizado en 2009 (Murrieta, 2009). En dicho estudio se clasifican las viviendas de las invasiones en cinco tipos:

En buen estado: son viviendas de al menos tres habitaciones que se encuentran terminadas.

Nivel intermedio: las que se encuentran en un proceso intermedio de construcción que pueden ser de uno o dos cuartos con otra pieza en proceso de construcción, con techos de concreto o lámina galvanizada y pisos de concreto.

En proceso-nivel precario: que constan de un cuarto de cartón o material de desecho, pero que al lado se construye una o más piezas de material (no precario) o pueden ser cuartos construidos a base de ladrillo o block, pisos de cemento o sin piso y techo de lámina galvanizada en mal estado o material de desecho y muros sin enjarres.

Vivienda precaria: la que está totalmente construida de lámina de cartón negro, madera o material de desecho y que no tiene los inicios de una construcción de material duradero (no precario).

Lote baldio: sin construcción y sin habitar.

De acuerdo con este estudio que comprendió la revisión visual y directa de las viviendas, se encontró que de un total de 18601 viviendas en 31 invasiones, el $45 \%$ de las viviendas se encontraba en buen estado, el 34 \% en un nivel intermedio, el $7 \%$ eran viviendas en proceso de nivel precario y el $8 \%$ corresponde a viviendas precarias. El resto correspondió a lotes baldíos (Murrieta, 2009). 


\begin{tabular}{|c|c|c|c|c|c|c|c|}
\hline & Invasión & Total de viviendas & $\begin{array}{l}\text { Viviendas en buen } \\
\text { estado }\end{array}$ & $\begin{array}{l}\text { Viviendas en } \\
\text { proceso-nivel } \\
\text { intermedio }\end{array}$ & $\begin{array}{l}\text { Viviendas en } \\
\text { proceso-nivel } \\
\text { precario }\end{array}$ & $\begin{array}{l}\text { Viviendas } \\
\text { precaria }\end{array}$ & Lote baldío \\
\hline 1 & Altares & 943 & 99 & 353 & 174 & 267 & 50 \\
\hline 2 & Amapolas & 749 & 407 & 278 & 18 & 22 & 24 \\
\hline 3 & Bella Vista & 307 & 100 & 175 & 15 & 13 & 4 \\
\hline 4 & Hacienda de la Flor & 258 & 103 & 117 & 15 & 16 & 7 \\
\hline 5 & Internacional & 636 & 212 & 371 & 21 & 26 & 6 \\
\hline 6 & Insurgentes & 2276 & 1710 & 456 & 43 & 51 & 16 \\
\hline 7 & Metalera & 445 & 292 & 111 & 3 & 25 & 14 \\
\hline 8 & Laura Alicia Frías & 1007 & 178 & 381 & 150 & 263 & 35 \\
\hline 9 & Adolfo López Mateos (norte) & 149 & 138 & 7 & 0 & 0 & 4 \\
\hline 10 & Apache & 1721 & 939 & 235 & 28 & 77 & 442 \\
\hline 11 & Café Combate & 738 & 62 & 304 & 202 & 105 & 65 \\
\hline 12 & $\begin{array}{l}\text { Cerro de la Campana } \\
\text { La Matanza y Las Pilas }\end{array}$ & 387 & 278 & 82 & 0 & 27 & 0 \\
\hline 13 & El Jito & 338 & 289 & 45 & 0 & 0 & 4 \\
\hline 14 & Héctor Francisco Romero & 72 & 18 & 32 & 15 & 6 & 1 \\
\hline 15 & $\begin{array}{l}\text { Nuevo Progreso } \\
\text { (cuartel La Lengüeta) }\end{array}$ & 160 & 51 & 59 & 11 & 22 & 17 \\
\hline 16 & Red 2000 (antiguo Basurón) & 182 & 22 & 64 & 14 & 79 & 3 \\
\hline 17 & $\begin{array}{l}\text { Machi López } \\
\text { (cuartel Progresista II) }\end{array}$ & 579 & 538 & 27 & 2 & 3 & 9 \\
\hline 18 & San Luis & 481 & 249 & 201 & 23 & 2 & 6 \\
\hline 19 & $\begin{array}{l}\text { Sol Aguilar } \\
\text { (cuartel El Triunfo) }\end{array}$ & 115 & 49 & 58 & 4 & 2 & 2 \\
\hline 20 & Tirocapes & 399 & 286 & 105 & 2 & 4 & 2 \\
\hline 21 & $\begin{array}{l}\text { Unión de Ladrilleros } \\
\text { (Luis Donaldo Colosio) }\end{array}$ & 198 & 106 & 47 & 9 & 8 & 28 \\
\hline 22 & Manuel Gómez Morín & 754 & 378 & 315 & 16 & 31 & 14 \\
\hline 23 & 4 de Marzo & 583 & 208 & 176 & 91 & 61 & 47 \\
\hline 24 & 4 Olivos & 134 & 46 & 78 & 7 & 2 & 1 \\
\hline 25 & Adolfo López Mateos (sur) & 1986 & 808 & 800 & 119 & 187 & 72 \\
\hline 26 & El Mirador & 471 & 114 & 274 & 31 & 28 & 24 \\
\hline 27 & $\begin{array}{l}\text { Francisco de Paula y } \\
\text { Solidaridad, V Etapa }\end{array}$ & 160 & 41 & 44 & 40 & 17 & 18 \\
\hline 28 & Ladrilleras & 1302 & 344 & 681 & 122 & 119 & 36 \\
\hline 29 & $\begin{array}{l}\text { Las Minitas } \\
\text { (sector Quiroga) }\end{array}$ & 117 & 35 & 64 & 6 & 5 & 7 \\
\hline 30 & Lomas del Norte y del Paraíso & 388 & 71 & 131 & 86 & 59 & 41 \\
\hline \multirow[t]{3}{*}{31} & Luis Donaldo Colosio & 566 & 131 & 308 & 70 & 34 & 23 \\
\hline & Totales & 18601 & 8302 & 6379 & 1337 & 1561 & 1022 \\
\hline & Porcentajes & $100 \%$ & $45 \%$ & $34 \%$ & $7 \%$ & $8 \%$ & $5 \%$ \\
\hline
\end{tabular}

Fuente: adaptado de América Murrieta del Programa Paso a Paso mi Casa, 2009 (Murrieta, 2009).

De este estudio se desprende primeramente que el proceso de autoconstrucción es muy dinámico y avanza de manera rápida en las invasiones. De acuerdo con información proporcionada, avanza más mientras mayor es la antigüedad de las invasiones. Por otra parte, se aprecia que existe un $15 \%$ de viviendas precarias que por su tipo de materiales de construcción, como lámina o cartón, no protegen a las familias de las inclemencias del tiempo. En 2009, el número de viviendas precarias era de 2898 , de acuerdo con este inventario.

Otro tema importante son los servicios públicos urbanos con que cuentan las viviendas de las invasiones. Con la colaboración de personal de Sindicatura Municipal (López, 2011), se hizo un inventario de los servicios disponibles en 30 invasiones dependientes de esa oficina y se obtuvo que, de ese total de invasiones, cinco no cuentan con energía eléctrica, tres con agua conectada al sistema de la ciudad, diez con conexión a la red drenaje sanitario y seis con alumbrado público. No obstante, es importante señalar que, en la mayoría de los casos, aunque dichos servicios no se encuentran disponibles de manera regular, los habitantes de las invasiones desarrollan diversas estrategias para contar con los mismos. 


\begin{tabular}{|c|c|c|c|c|c|c|c|}
\hline & Invasión & $\begin{array}{l}\text { Energía } \\
\text { eléctrica }\end{array}$ & $\begin{array}{l}\text { Agua } \\
\text { potable }\end{array}$ & Drenaje & Pavimento & $\begin{array}{l}\text { Alumbrado } \\
\text { público }\end{array}$ & $\begin{array}{l}\text { Cobertura } \\
\text { aprox. }\end{array}$ \\
\hline 1 & Miguel Ángel Jiménez & Sí & Sí & 0 & 0 & Sí & $60 \%$ \\
\hline 2 & Héctor Francisco Romero & Sí & Sí & & & Sí & $60 \%$ \\
\hline 3 & El Triunfo (Sol Aguilar) & Sí & Sí & Sí & & Sí & $80 \%$ \\
\hline 4 & Combate & Sí & Sí & Sí & & Sí & $80 \%$ \\
\hline 5 & San Luis & Sí & Sí & Sí & & Sí & $80 \%$ \\
\hline 6 & Las Palmas & Sí & Sí & Sí & Sí & Sí & $100 \%$ \\
\hline 7 & Progresista & Sí & Sí & Sí & & Sí & $80 \%$ \\
\hline 8 & Nueva Victoria & Sí & Sí & & & Sí & $60 \%$ \\
\hline 9 & Insurgentes CTM & Sí & Sí & Sí & & Sí & $80 \%$ \\
\hline 10 & Unión de Ladrilleros & Sí & Sí & Sí & & Sí & $80 \%$ \\
\hline 11 & Miguel Valencia & & & & & & $0 \%$ \\
\hline 12 & Red 2000 & & & & & & $0 \%$ \\
\hline 13 & El 33 & & Sí & & & & $20 \%$ \\
\hline 14 & Guayacán & & & & & & $0 \%$ \\
\hline 15 & Insurgentes Barboza & Sí & Sí & & & Sí & $60 \%$ \\
\hline 16 & Adolfo López Mateos & Sí & Sí & Sí & & Sí & $80 \%$ \\
\hline 17 & Apache & Sí & Sí & Sí & Sí & Sí & $100 \%$ \\
\hline 18 & Ampliación Ladrilleras & Sí & Sí & Sí & Sí & & $80 \%$ \\
\hline 19 & Solidaridad IV & Sí & Sí & Sí & Sí & Sí & $100 \%$ \\
\hline 20 & El Mirador & Sí & Sí & Sí & Sí & Sí & $100 \%$ \\
\hline 21 & 4 de Marzo & Sí & Sí & Sí & Sí & & $80 \%$ \\
\hline 22 & $\begin{array}{l}\text { Lomas del Norte y del } \\
\text { Paraíso }\end{array}$ & Sí & Sí & Sí & Sí & Sí & $100 \%$ \\
\hline 23 & Luis Donaldo Colosio III & Sí & Sí & Sí & Sí & Sí & $100 \%$ \\
\hline 24 & Altares & & Sí & & & Sí & $40 \%$ \\
\hline 25 & Arco Iris & Sí & Sí & & & Sí & $60 \%$ \\
\hline 26 & Bella Vista & Sí & Sí & Sí & & Sí & $80 \%$ \\
\hline 27 & Hacienda de la Flor & Sí & Sí & Sí & & Sí & $80 \%$ \\
\hline 28 & Hermosa Provincia & Sí & Sí & Sí & & Sí & $80 \%$ \\
\hline 29 & Insurgentes & Sí & Sí & Sí & & Sí & $80 \%$ \\
\hline \multirow[t]{3}{*}{30} & Internacional & Sí & Sí & Sí & & Sí & $80 \%$ \\
\hline & Totales & 25 & 27 & 20 & 8 & 24 & \\
\hline & Porcentaje & $83 \%$ & $90 \%$ & $67 \%$ & $27 \%$ & $80 \%$ & \\
\hline
\end{tabular}

Fuente: elaborado con base en reporte de Óscar López Salido, Análisis de invasiones atendidas por Sindicatura Municipal, H. Ayuntamiento de Hermosillo, 2011.

En lo que respecta a energía eléctrica, por ejemplo, los habitantes de las colonias se proveen por medio de conexiones precarias consistentes en cables que ellos mismos "cuelgan" de los transformadores o del alumbrado público y que son fáciles de observar en las invasiones que carecen de este servicio.

Asimismo, en lo que respecta a la disponibilidad de agua, el gobierno municipal suple la deficiencia por medio de un servicio de entrega de agua por medio de carros cisterna, conocidos como "pipas", que les entregan agua de manera periódica y gratuita. Por último, la carencia de drenaje generalmente es cubierta con fosas, lo que se conoce como "excusados de cajón" o, en algunos casos, con el fecalismo.

En lo que respecta a la cobertura de los servicios, destaca que hay tres invasiones que no cuentan absolutamente con servicios: Miguel Valencia (una parte), Red 2000 y Guayacán. Son colonias en las que no se ha emprendido la negociación 
para la adquisición del terreno o que, por circunstancias como la de estar asentadas en un antiguo tiradero de basura, no son susceptibles de ser regularizadas, y por lo mismo, no se les ha proveído de ningún servicio. Por otra parte, hay dos invasiones, El 33 y Altares, que cuentan con un bajo nivel de cobertura de servicios. Estas cinco invasiones parecen ser las que concentran el mayor grado de precariedad y vulnerabilidad.

Cuadro 4. Asentamientos con mayor carencia de servicios urbanos

\begin{tabular}{ccccc}
\hline Invasión & Superficie & Familias & Servicios & Agua por pipas \\
\hline Miguel Valencia & 8 ha & 365 & Ninguno & Sí \\
Red 2000 & 3 ha & 180 & Ninguno & Sí \\
Guayacán & n.d. & 750 & Ninguno & Sí \\
El 33 & $4000 \mathrm{~m}^{3}$ & 40 & Agua potable y alumbrado & Sí \\
Altares* & n.d. & n.d. & n.d. & Sí \\
Total & & 1335 & & \\
\hline
\end{tabular}

Fuente: elaborado con base en reporte de Óscar López Salido, Análisis de invasiones atendidas por Sindicatura Municipal, H. Ayuntamiento de Hermosillo, 2011.

* La regularización de la invasión Altares corresponde a la Comisión Estatal de Bienes y Concesiones.

Las invasiones más vulnerables por su falta de servicios están habitadas por 1335 familias (sin incluir la invasión Altares) que equivalen aproximadamente a 5340 personas que, por las precarias condiciones en que viven, están en grave riesgo durante el verano.

\section{Características de la vivienda y su población}

Como parte de esta investigación se levantó una encuesta en 10 invasiones o asentamientos irregulares que comprendió a 365 viviendas y a 1199 habitantes de dichas viviendas (Sindicatura Municipal de Hermosillo, 2010). El levantamiento de la encuesta se hizo entre enero y noviembre de 2010 por el personal de la Sindicatura Municipal, y se utilizó el Cuestionario Único de Información Socioeconómica (CUIS), utilizado por la Secretaría de Desarrollo Social (SEDESOL, 2010), al que se le agregaron las dos preguntas finales relacionadas directamente con la vulnerabilidad al clima. A continuación se presenta el resumen de las respuestas obtenidas en cada tema:

Pavimentación: con respecto al entorno urbano en que se ubican estos asentamientos, tenemos que solo el $13 \%$ de las viviendas se ubica en calles pavimentadas, mientras que las restantes se localizan en calles sin pavimento. Además, el $89 \%$ de las viviendas no cuenta con banqueta, aunque el $82 \%$ reportó que cuenta con alumbrado público.

Propiedad y pago de la vivienda: el 63 \% reporta que no tiene escrituras de la casa. El $28 \%$ reporta que está pagando la casa y el 27 declara que es propia y está totalmente pagada.

Condiciones de la vivienda: prácticamente todas las viviendas son independientes y unifamiliares, es decir, no se trata de vecindades ni comunidades de más de una familia. El $60 \%$ de las viviendas tiene solo uno o dos cuartos. E1 $25 \%$ tiene tres cuartos y el $14 \%$ restante tiene cuatro habitaciones o más. El $65 \%$ de las viviendas tiene un solo dormitorio o cuarto que se usa para dormir. Esto significa que en algunas familias con hijos u otros dependientes, toda la familia duerme en la misma habitación. En el $24 \%$ de los casos, se cocina y se duerme en la misma habitación.

Materiales de la vivienda: el $25 \%$ de las viviendas tiene piso de tierra. Existe un $26 \%$ de viviendas que tiene techos precarios como lámina de cartón, metálica, de asbesto o de desecho. Las restantes tienen techo de concreto o de vigueta con bovedilla. Existe un $15 \%$ de viviendas cuyos muros son de cartón, lámina metálica, madera o de desecho. Las restantes son de ladrillo, block o tabique.

Servicios sanitarios: solo el $46 \%$ de las viviendas tiene conexión al drenaje y está en posibilidad de usar los sanitarios convencionales que desalojan los desechos con base en flujos de agua. El $42 \%$ de las viviendas no cuenta con sanitario convencional y recurre a pozos, hoyos, letrinas secas o húmedas. Un $3 \%$ no cuenta con ningún tipo de sanitario y se puede inferir que recurre al fecalismo, es decir, "hace sus necesidades" a la intemperie. Casi todas las viviendas que cuentan con baño lo tienen exclusivo para la vivienda y no recurren a baños compartidos o colectivos.

Suministro de agua: el $1 \%$ de las casas la obtiene por medio de las pipas del ayuntamiento. El $46 \%$ por medio de una toma ubicada fuera de su vivienda, a través de una manguera o acarreándola. El $51 \%$ de las viviendas tiene agua conectada al sistema de la ciudad.

Energía eléctrica: un $8 \%$ de viviendas reporta que no tiene energía eléctrica. Otro $4 \%$ reporta que obtiene la energía eléctrica de "otra fuente" que no es el servicio público. E1 84 \% reporta que cuenta con servicio público de energía eléctrica.

Aparatos eléctricos: solo el $84 \%$ de las viviendas reporta que cuenta con refrigerador. El $42 \%$ tiene máquina lavadora.

\section{Savia}


El $14 \%$ cuenta con un automóvil o vehículo de transporte. E1 $5 \%$ reporta que tiene computadora.

Hacinamiento: el 11 $\%$ de las viviendas tiene seis habitantes o más. El $34 \%$ tiene cuatro o cinco habitantes y el $35 \%$ de las viviendas tiene dos o tres habitantes. En un $21 \%$ de las viviendas habita una sola persona.

En la gran mayoría de los casos, los habitantes de las viviendas comparten los gastos y también los alimentos. Algunas viviendas aparecen sin respuesta en estos rubros debido a que su situación no encajaba exactamente en las respuestas, pero está claro de que se trata de grupos familiares. Igualmente, casi todos los habitantes (96\%) viven regularmente en la vivienda y no se trata de gente que viva temporalmente o solo en fines de semana.

Población de las viviendas: el 53 \% de la población son hombres y el resto mujeres, o sea que hay más hombres. Este predominio de la población masculina se aprecia en todos los rangos de edad. De esto se puede inferir que, contrario a lo que sucede en el conjunto de la población, en las invasiones tiende a haber más hombres que mujeres.

En lo que se refiere a las edades de los habitantes de las viviendas, se encontró que el $20 \%$ de la población corresponde a niños menores de 10 años. E1 $23 \%$ corresponde a adolescentes y jóvenes de entre 11 y 20 años. La población adulta de entre 21 y 60 años de edad representa el $49 \%$ de los habitantes. La población mayor de 60 años constituye solo el $6 \%$. Aparentemente se trata de una población más joven que el conjunto de la población de la ciudad.

Lugar de origen y lengua: el $85 \%$ son originarios del estado de Sonora, el $4 \%$ de Sinaloa, el $1 \%$ de Chihuahua y el $10 \%$ restante son originarios de otros estados de la república, entre los que destacan la Ciudad de México, Oaxaca, Veracruz y Nayarit. El 3 \% habla una lengua indígena al igual que un $3 \%$ no habla español.

Seguridad social y discapacidades: el $27 \%$ de la población no está adscrita a ningún sistema y no cuenta con seguridad social y el $14 \%$ de la población tiene algún tipo de discapacidad.

Educación: un $8 \%$ de la población no es capaz de escribir un recado, es decir, no sabe leer ni escribir. E1 $35 \%$ de los habitantes asiste actualmente a la escuela. Los que la abandonaron lo hicieron principalmente por motivos económicos, para ayudar en su casa o porque no tienen dinero para solventar los costos del estudio.

Trabajo e ingresos: un $11 \%$ no trabaja o está buscando

trabajo. En esta pregunta hubo un $16 \%$ de no respuestas, y corresponden a aquellos cuya situación no se refleja en las opciones de respuesta y que puede interpretarse como correspondiente a trabajos informales o que son menores de edad. Por ello, en otra forma de la misma pregunta aparece que el $30 \%$ no trabaja. En este caso, las personas que no respondieron, al parecer son menores de edad. Entre los oficios más frecuentes destacan los de albañil (7 \%), obrero (5\%) y empleada doméstica (4\%), aunque la gran mayoría no se identificó con ninguna de las categorías del cuestionario. El promedio de ingreso mensual reportado es de $\$ 3728$ pesos. El ingreso más frecuente es de 4000 pesos al mes.

Nivel de marginación: el 29 \% reporta que en el último mes, al menos algún miembro de la familia no hizo una de las comidas del día por falta de dinero. El $24 \%$ reporta que incluso dejó de comer en todo un día por falta de recursos. Al menos el $10 \%$ reporta que algún miembro de la familia se fue a trabajar a otra localidad, otro estado o incluso fuera del país. Pero solo cinco viviendas $(1 \%)$ reportaron que reciben dinero de fuera del país. El $82 \%$ de las viviendas reporta que no recibe ningún apoyo de programas oficiales. E1 $10 \%$ recibe apoyos del programa Oportunidades o leche Liconsa.

Vulnerabilidad al clima: uno de los propósitos principales de la encuesta levantada era medir la vulnerabilidad al clima y más específicamente a las altas temperaturas. Para ello se hicieron preguntas sobre la disponibilidad de aparatos eléctricos como ventiladores, enfriadores de aire y aparatos de aire acondicionado; también sobre la incidencia de enfermedades relacionadas con la exposición al calor.

Equipamiento contra el calor: el $3 \%$ de las viviendas no cuenta con ningún tipo de aparato eléctrico para mitigar las altas temperaturas. Un $61 \%$ de las viviendas tiene al menos un enfriador de aire (cooler o aerocooler). Existe un $28 \%$ de viviendas con al menos un aparato de aire acondicionado. 


\section{Al menos un aire acondicionado}

\section{Al menos un enfriador de aire}
Al menos un ventilador

Ningún

aparato

Total de viviendas (328)

91

$28 \%$
199

$61 \%$

196

$\%$

Fuente: elaboración con datos de la encuesta levantada por Sindicatura Municipal y El Colegio de Sonora entre enero y noviembre de 2010.

Incidencia de deshidratación: 143 personas o $12 \%$ de los habitantes de las invasiones padeció deshidratación en 2010. El $58 \%$ de los deshidratados fueron hombres. Los niños menores de 12 años se deshidrataron el doble que los adultos.

Cuadro 6. Personas que padecieron deshidratación en 2010

\begin{tabular}{|c|c|c|}
\hline Indicador & Valor absoluto & Porcentaje \\
\hline $\begin{array}{l}\text { Total de personas enfermas por calor o } \\
\text { deshidratación en el año } 2010\end{array}$ & 143 & $\begin{array}{l}12 \% \text { (con respecto a } 1200 \\
\text { personas) }\end{array}$ \\
\hline $\begin{array}{l}\text { Número de viviendas con persona(s) } \\
\text { enferma(s) por calor o deshidratación } \\
\text { en el año } 2010\end{array}$ & 88 & $\begin{array}{l}27 \% \text { (con respecto a } 328 \\
\text { viviendas) }\end{array}$ \\
\hline
\end{tabular}

Fuente: elaboración con datos de la encuesta levantada por Sindicatura Municipal y El Colegio de Sonora entre enero y noviembre de 2010.

Figura 1. Deshidratación por edades

Sexo de la persona afectada por calor

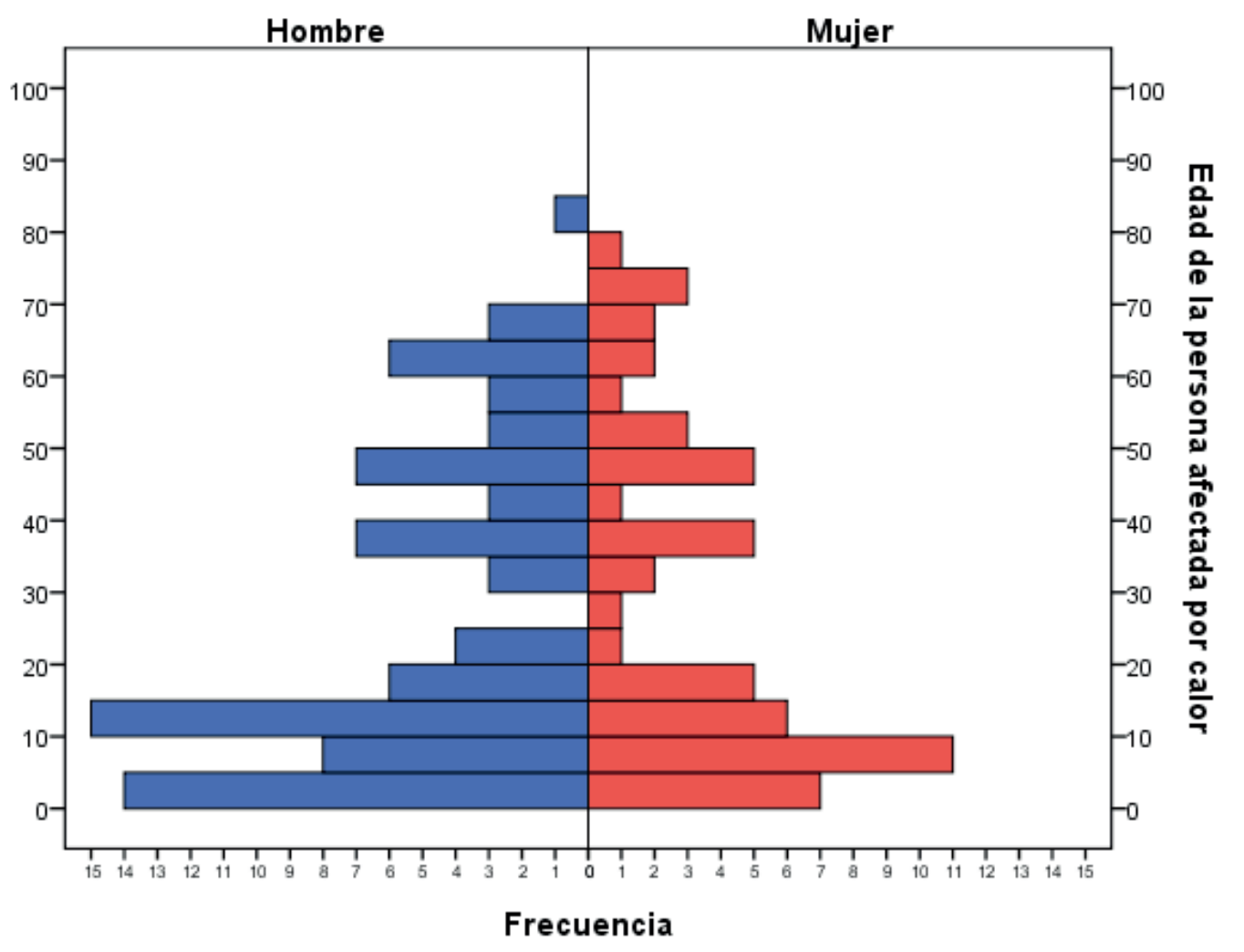

Fuente: elaboración con datos de la encuesta levantada por Sindicatura Municipal y El Colegio de Sonora entre enero y noviembre de 2010. 
Se requiere más investi-gación a fin de ponderar estos porcentajes y determinar si son significativamente más altos que los de la población en general. Sin embargo, partiendo del criterio de que, dadas las condiciones climáticas de la ciudad, ninguna vivienda debería de carecer de equipamiento para mitigar las altas temperaturas; es un dato relevante que existan viviendas que no cuentan con equipos para este propósito. De la misma manera, el criterio general es que nadie debería de padecer deshidratación. Por lo tanto, las cifras y porcentajes mostrados corresponden a situaciones que no deberían de existir.

\section{Conclusiones}

Por la información revisada y por los datos recabados se aprecia que existe una porción significativa de la población de Hermosillo que se puede considerar vulnerable al clima y sobre la cual deben de hacerse previsiones a fin de reducir su nivel de vulnerabilidad. De acuerdo con la información recabada por medio de Sindicatura y del "Programa Paso a Paso mi Casa", se observó que en los asentamientos irregulares conocidos como invasiones habita población que puede ser considerada como vulnerable al clima. En 2010 había 70 invasiones en la ciudad en las que vivían más de 6000 familias, es decir, aproximadamente 25000 personas.

En una muestra de 31 invasiones se observó que el $15 \%$ de las viviendas son precarias. Se trata de casi 1900 viviendas tan solo en estas invasiones (la mitad de las existentes) cuyas condiciones no permiten a sus habitantes protegerse de las inclemencias del tiempo.

Asimismo, al revisar la disponibilidad de servicios, se detectó que cinco invasiones no cuentan con energía eléctrica, tres con agua conectada al sistema de la ciudad y 10 con conexión a la red de drenaje sanitario. Estos son los asentamientos más vulnerables de Hermosillo por su grado de exposición al calor y a las inclemencias del tiempo: Miguel Valencia, Red 2000, Guayacán, El 33 y la invasión Altares, las cuales carecen de los servicios básicos de agua o energía eléctrica, o de ambos. En estas invasiones habitan alrededor de 1500 familias, es decir, aproximadamente 6 000 personas que se encuentran en riesgo severo de sufrir deshidrataciones o golpes de calor. Además, su nivel de vida es precario por estar expuestos a las inclemencias del tiempo.

Igualmente, en la encuesta levantada en diez asentamientos irregulares, se observó alta frecuencia de techos y muros precarios, altos niveles de hacinamiento y un alto número de viviendas que carecen de agua conectada al sistema de la ciudad, energía eléctrica o drenaje. En lo que respecta a las personas que viven en las invasiones, se observó una mayor proporción de hombres que de mujeres, así como una gran población infantil y juvenil. Además, existe una baja escolaridad y un gran desempleo. Se destaca también, la carencia de apoyos de los programas oficiales, la no adscripción a los sistemas de seguridad social y la frecuencia de incapacidades. Como era de esperarse, los ingresos están por debajo de la línea de pobreza y una parte de las familias reporta que ha dejado de comer por falta de recursos.

En lo que respecta a la vulnerabilidad al calor, se observó que existen 11 viviendas que no cuentan con ningún tipo de aparato eléctrico que les ayude a mitigar las altas temperaturas; también que hay una parte de las viviendas que solo cuenta con ventiladores eléctricos, los cuales, en los días calurosos, no son suficientes para mitigar el calor, ya que arrojan aire caliente.

La encuesta levantada muestra que el $12 \%$ de las personas de los asentamientos irregulares sufrió alguna deshidratación en 2010 y que este porcentaje corresponde principalmente a niños y niñas menores de 12 años.

En general, se aprecia que existe una porción significativa de la población de Hermosillo que es altamente vulnerable al calor y a las temperaturas extremas; por ello, se requiere de una atención y de programas especiales que los ayuden a reducir su vulnerabilidad y a superar esta condición. Estos programas deberán atender tanto las condiciones de la vivienda como los niveles de ingreso y equipamiento de sus habitantes. 


\begin{tabular}{|c|c|c|c|c|}
\hline Núm. & A cargo de Sindicatura & $\begin{array}{l}\text { Año de } \\
\text { origen }\end{array}$ & $\begin{array}{l}\text { No. de } \\
\text { familias }\end{array}$ & Ubicación \\
\hline 1 & Miguel Valencia & 2002 & 250 & Blvd. Progreso y Solidaridad \\
\hline 2 & Plutarco Elías Calles & 2008 & 230 & Norponiente de la ciudad \\
\hline 3 & Insurgentes Construtécnica & 1990 & 400 & Norponiente de la ciudad \\
\hline 4 & Insurgentes C.T.M. & 1990 & 104 & Mesa del Seri y Blvd. Las Torres \\
\hline 5 & Nueva Victoria & 1998 & 180 & La Victoria \\
\hline 6 & Nuevo Amanecer & 1995 & 85 & Norponiente de la ciudad \\
\hline 7 & Ampliación Sol Aguilar & 1999 & 60 & Norte de la ciudad \\
\hline 8 & San Luis & 1990 & 80 & Salida norte de la ciudad \\
\hline 9 & Héctor Fco. Romero* & 1998 & 65 & Blvd. Lázaro Cárdenas, norte de la ciudad \\
\hline 10 & El 33 & 1996 & 40 & Olivares final, norte de la ciudad \\
\hline 11 & $\begin{array}{l}\text { La Alborada Sección } \\
\text { İñiguez }\end{array}$ & 1992 & 25 & Blvd. Lázaro Cárdenas, norte de la ciudad \\
\hline 12 & Guayacán & 2008 & 750 & Reforma final, norte de la ciudad \\
\hline 13 & Unión de ladrilleros* & 1990 & 170 & Norponiente de la ciudad \\
\hline 14 & Francisco Villa & 1987 & 45 & Norte de la ciudad \\
\hline 15 & Adolfo López Mateos & 1994 & 1200 & Salida sur de la ciudad \\
\hline 16 & Las Palmas & 1998 & 40 & Progreso y Reforma \\
\hline 17 & Elmo Forte & & 20 & Salida norte de la ciudad \\
\hline 18 & Tiro al Blanco & & 40 & Sur de la ciudad \\
\hline 19 & Las Taparitas & & 200 & Norponiente de la ciudad \\
\hline 20 & Miguel Ángel Jiménez & 1998 & 50 & Norte de la ciudad \\
\hline 21 & Machi López* & 1998 & 250 & Norte de la ciudad \\
\hline 22 & Sol Aguilar* & & 200 & Norte de la ciudad \\
\hline 23 & El Chaparral & 2004 & 70 & Norte de la ciudad \\
\hline
\end{tabular}

Núm. A cargo de Promotora Inmobiliaria

\begin{tabular}{|c|c|c|c|c|}
\hline 1 & La Manga sector cota & 2003 & 41 & Poniente de la ciudad \\
\hline 2 & La Alborada (las ladrilleras) & 2002 & 10 & Norte de la ciudad \\
\hline 3 & Luis Donaldo Colosio IV etapa & 1995 & 177 & Norponiente de la ciudad \\
\hline 4 & Ladrilleras (Siria Campa) & & 78 & Norte de la ciudad \\
\hline 5 & $\begin{array}{l}\text { Ampliación Ladrilleras (Romo } \\
\text { Pablos) }\end{array}$ & 1997 & 50 & Norte de la ciudad \\
\hline 6 & 4 Olivos Margaritas & 1996 & 109 & Norponiente de la ciudad \\
\hline 7 & La Plancha & 1993 & 150 & Norponiente de la ciudad \\
\hline 8 & Solidaridad Occidental & 2003 & 789 & Norponiente de la ciudad \\
\hline 9 & Francisco Villa & 1980 & 40 & Norte de la ciudad \\
\hline 10 & La Ponderosa & 1997 & 171 & Norte de la ciudad \\
\hline 11 & Las Carretas & 1990 & 150 & Sur de la ciudad \\
\hline
\end{tabular}

Año de origen 


\begin{tabular}{clll}
\hline 1 & Adolfo de la Huerta & 16 & Nuevo Horizonte \\
2 & Altares & 17 & Palo Verde Indeur \\
3 & Amapolas & 18 & Primero Hermosillo \\
4 & Arcoíris & 19 & Renacimiento \\
5 & Bella Vista & 20 & Sahuaro Indeco \\
6 & Constitución & 21 & Bienes y Concesiones \\
7 & Eusebio Kino & 22 & Ampliación Progreso \\
8 & Hacienda de la Flor & 23 & Bienes y Concesiones \\
9 & Hermosa Provincia & 24 & Tojungo \\
10 & Insurgentes & 25 & El Chaparral \\
11 & Internacional & 26 & Bienes y Concesiones \\
12 & Mártires de Cananea & 27 & Ampliación Altares \\
13 & Metalera & 28 & Camorca \\
14 & Mirasoles & 29 & Realito \\
15 & Nueva Esperanza & & \\
\hline \hline
\end{tabular}

\section{A cargo de CORETT}

\begin{tabular}{llll}
\hline \hline 1 & Akihuiki & 6 & Tiro al Blanco \\
2 & Apache (una parte) & 7 & Villa de Seris (una parte) \\
3 & Minitas (una parte) & 8 & Villa Hermosa \\
4 & Palo Verde (una parte) & 9 & Y Griega \\
5 & Pedregal de la Villa (una parte) & & \\
\hline
\end{tabular}

\section{Invasiones regularizadas por Sindicatura (históricas)}

\begin{tabular}{|c|c|c|c|c|c|}
\hline 1 & 4 de Marzo & 30 & Eা Mariachা & 59 & Machi López" \\
\hline 2 & 4 Olivos & 31 & El Ranchito & 60 & Manuel Gómez Morín \\
\hline 3 & 5 de Mayo & 32 & El Torreón & 61 & Mariachi \\
\hline 4 & Adolfo de la Huerta & 33 & El Triunfo & 62 & Miguel Hidalgo \\
\hline 5 & Álvaro Obregón & 34 & Emiliano Zapata I & 63 & Miguel Ángel Jiménez \\
\hline 6 & Ángel Flores & 35 & Emiliano Zapata II & 64 & Modelo \\
\hline 7 & Apache & 36 & Ferrocarril & 65 & acameri (una parte) \\
\hline 8 & Álamos & 37 & Fonhapo & 66 & Nuevo Progreso \\
\hline 9 & Bahía de Kino & 38 & Heberto Castillo & 67 & Norberto Ortega \\
\hline 10 & Balderrama & 39 & Héctor Fco. Romero* & 68 & Olivares \\
\hline 11 & Benito Juárez & 40 & Inalámbrica & 69 & Palo Verde (E. Zapata) \\
\hline 12 & Café Combate & 41 & Insurgentes (una parte) & 70 & Pimentel \\
\hline 13 & Calle 12 & 42 & Jacinto López & 71 & PRI Hermosillo \\
\hline 14 & Camino Real & 43 & Jesús García & 72 & Progresista \\
\hline 15 & Canal Chanate & 44 & La Lengüeta & 73 & Quinta Emilia \\
\hline 16 & Canal Pitahayita & 45 & La Manga & 74 & Red 2000 \\
\hline 17 & Cañada de los Negros & 46 & La Matanza & 75 & Ranchito \\
\hline 18 & Carmen Serdán & 47 & Las Cuevas & 76 & Rinconada de la Cruz \\
\hline 19 & Centro (una parte) & 48 & Las Ladrilleras & 77 & Rinconada Nuevo León \\
\hline 20 & Coloso Alto & 49 & Las Palmas & 78 & Rosario I. de Piedra \\
\hline 21 & Cerro de la Campana & 50 & Las Pilas & 79 & Sahuaro Municipal \\
\hline 22 & Choyal & 51 & Ley 57 & 80 & San Benito \\
\hline 23 & Club Hípico & 52 & Libertad & 81 & San Luis \\
\hline 24 & Cuartel Pitic & 53 & Loma Linda (una parte) & 82 & Santa Isabel \\
\hline 25 & Cuartel Zona & 54 & Lomas de Madrid & 83 & Sector Beltrones \\
\hline
\end{tabular}




\begin{tabular}{|c|c|c|c|c|c|}
\hline 26 & División del Norte & 55 & Lomas de Pitic & 84 & Sol Aguilar* \\
\hline 27 & El Jito & 56 & López Portillo & 85 & Tirocapes \\
\hline 28 & El Llano & 57 & Los Álamos & 86 & Unión de Ladrilleros* \\
\hline 29 & El Malecón & 58 & Luis Encinas & 87 & Villa de Seris (una parte) \\
\hline
\end{tabular}

\begin{tabular}{|c|c|c|c|c|}
\hline Núm & $\begin{array}{l}\text { Invasiones regularizadas por Promotora } \\
\text { Inmobiliaria (históricas) }\end{array}$ & Año & No. Familias & Ubicación \\
\hline 1 & Lomas del Norte y del Paraíso & 2007 & 386 & Norte de la ciudad \\
\hline 2 & Ladrilleras (Perla del sol y Symonds) & 2006 & 52 & Norte de la ciudad \\
\hline 3 & El Mirador & 2003 & 339 & Norponiente de la ciudad \\
\hline 4 & Ampliación El Mirador (Mancilla) & 2003 & 123 & Norponiente de la ciudad \\
\hline 5 & Solidaridad $V$ etapa bis & 2002 & 83 & Norponiente de la ciudad \\
\hline 6 & Los Mirasoles & 2002 & 20 & Poniente de la ciudad \\
\hline 7 & Solidaridad Polígono "J" & 2000 & & Norponiente de la ciudad \\
\hline 8 & Héctor Fco. Romero & 1998 & 18 & Norponiente de la ciudad \\
\hline 9 & Ampliación Ladrilleras & 1997 & 660 & Norte de la ciudad \\
\hline 10 & Café Combate & 1997 & 178 & Norte de la ciudad \\
\hline 11 & 4 de Marzo & 1997 & 528 & Norte de la ciudad \\
\hline 12 & Solidaridad IV etapa (Mendía) & 1996 & 528 & Norponiente de la ciudad \\
\hline 13 & Sahuaro final municipal & 1995 & 431 & Poniente de la ciudad \\
\hline 14 & Luis Donaldo Colosio III etapa & 1995 & 273 & Norponiente de la ciudad \\
\hline 15 & Solidaridad III etapa & 1993 & 328 & Norponiente de la ciudad \\
\hline 16 & Solidaridad V etapa & 1993 & 77 & Norponiente de la ciudad \\
\hline 17 & Solidaridad I & 1992 & 1053 & Norponiente de la ciudad \\
\hline 18 & Solidaridad B & 1992 & 940 & Norponiente de la ciudad \\
\hline 19 & Las Minitas Sec. Las Rosas y Miravalles & 1995 y 1999 & 757 & Surponiente de la ciudad \\
\hline 20 & Solidaridad II etapa bis & 1992 y 1993 & 825 & Norponiente de la ciudad \\
\hline 21 & Ladrilleras PIMH & & 242 & Norte de la ciudad \\
\hline 22 & Solidaridad Fco. de Paula García & & 34 & Norponiente de la ciudad \\
\hline 23 & Ampliación El Apache & & 118 & Sur de la ciudad \\
\hline 24 & San Judas Tadeo & & 68 & Norponiente de la ciudad \\
\hline 25 & Ampliación Las Palmas & & 63 & Norte de la ciudad \\
\hline 26 & Progresista & & 6 & Norte de la ciudad \\
\hline
\end{tabular}

Fuente: listado proporcionado por Sindicatura Municipal en mayo 2010.

\section{Bibliografía}

Gutiérrez, J.R. (1994). Los barrios de Hermosillo. Hermosillo, Sonora: Edición del autor.

Lagarda Lagarda, I. (2008). Historia de Hermosillo, origen, fundo legal, antiguos ejidos. Hermosillo, Sonora: Ayuntamiento de Hermosillo.

López Salido, O.A. (2011). Análisis de invasiones atendidas por Sindicatura Municipal. Presentación, Ayuntamiento de Hermosillo.

López Salido, O.A. (2010). Recuadro del Plano PL-Hillo-A1-01 Asentamientos Irregulares 2010. Mapa urbano con recuadro de información, Hermosillo: Sindicatura Municipal.

Martínez, Rascón, C. (2002). Los avecindados ejidales como nuevos actores sociales de la periferia hermosillense. En G. García Figueroa, N. Pineda Pablos, S.A. Sandoval Godoy, P. Wong González, J. Enríquez Acosta y A. Fuentes Fierro (coord.). Sonora frente al Siglo XXI (329-354). Hermosillo, Sonora: E1 Colegio de Sonora.
Méndez Sáinz, E. (2000). Hermosillo en el siglo XX. Urbanismos incompletos y arquitecturas emblemáticas. Hermosillo, Sonora: E1 Colegio de Sonora.

Murrieta Saldivar, A. (2009). Diagnóstico de las viviendas precarias en invasiones de Hermosillo. Programa Paso a Paso mi Casa. Estudio no publicado, Hermosillo, Sonora.

Secretaría de Desarrollo Social (SEDESOL). (2010). Programa de apoyo a los avecindados en condiciones de pobreza patrimonial para regularizar asentamientos humanos irregulares (PASPRAH). Recuperado de http://www.2006-2012.sedesol.gob.mx/ es/SEDESOL/Evaluacion_en_materia_de_Diseno_del_ PASPRAH

Sindicatura Municipal de Hermosillo. (2010). Encuesta sobre vivienda precaria y grupos vulnerables al clima en la ciudad de Hermosillo, Sonora. Reporte, Hermosillo, Sonora: El Colegio de Sonora.

Varady, R.G., C.A. Scott, M. Wilder, B. Morehouse, N. Pineda, \& G.H. Garfin. (2013). Transboundary adaptive management to reduce climate-change vulnerability in the Western U.S.-Mexico border region. Environmental Science and Policy 26 (Feb.). Special Issue, 102-112.

\section{Savía}

